\title{
On the Theory of Structuring Magnetic Suspensions
}

\author{
A. Yu. Zubarev and V. O. Kostenko \\ Ural Federal University, pr. Lenina 51, Yekaterinburg, 620083 Russia
}

Received February 13, 2012

\begin{abstract}
The results of the three-dimensional computer and analytical simulation are presented for the kinetics of chain-shaped aggregate growth in suspensions of magnetizable non-Brownian particles. The results of the computer experiment show that, when the volume fraction of particles is no larger than 2-3\%, chain-shaped aggregates are formed in the suspensions under the action of a field. The dependence of average number $\left\langle n>\right.$ of particles in a chain on time $t$ is adequately described by the power law $\langle n\rangle=C t^{k}$. The experiment indicates that, in contract to the common power approximations, in which exponent $k$ is considered to be a universal constant parameter, it depends on the concentration of particles and their interactions with walls bounding a suspension. At concentrations noticeably exceeding $2-3 \%$, dense bulk aggregates are formed in suspensions. The kinetics of their growth depends on the sizes of a suspension-containing vessel.
\end{abstract}

DOI: $10.1134 / \mathrm{S} 1061933 \mathrm{X} 13010183$

\section{INTRODUCTION}

Suspensions of microsized magnetizable particles in nonmagnetic liquids (magnetorheological suspensions (MRSs)) are attracting great interest from researchers and practitioners due to their unique properties that are realized in many modern high technologies. Reviews devoted to the physics of magnetic suspensions and the fields of their practical application may be found in $[1,2]$.

In the absence of magnetic fields, magnetic suspensions exhibit the behavior of ordinary suspensions of solid particles. Under the action of a magnetic field, the particles of these suspensions are magnetized and form various heterogeneous structures, predominantly linear chains and dense columns. The appearance of these structures and aggregates dramatically changes the physical properties of magnetic suspensions.

The methods and fields of application of MRSs in modern technologies are determined by the characteristic time of changes in their properties in an external field, this time is, in turn, governed by the times of the growth and breakage of internal structures. Therefore, the study of the growth kinetics of heterogeneous aggregates in magnetic suspensions is a problem of both practical and fundamental importance.

The first model of the evolution kinetics of chain aggregates in suspensions of magnetizable particles seems to have been proposed by Doi et al. [3]. At a given time moment, all aggregates of this hierarchical model have the same sizes, this, of course, being a rather strong simplification.

A number of computer models and experiments have showed the following power dependence of average number $\langle n\rangle$ of particles in chain-shaped aggregates of MRS on time $t:\langle n\rangle \sim t^{k}$, with exponent $k$ being sometimes considered to be some universal constant, the value of which lies in the range of $0.5-0.7[4,5]$. An attempt to theoretically substantiate this power law was made in [6]. Note that, in all these works, the Brownian motion of nanoparticles was ignored. A model of the aggregation of Brownian particles into linear chains was developed in [7] based on the Smoluchowski kinetic equation for the chain size distribution function. The results obtained in terms of model [7] are in good agreement with experimental data. However, this model implies that Brownian effects dramatically influence the kinetics of chain formation. At the same time, the Brownian phenomena in magnetic suspensions are, in many cases, strongly suppressed, because the magnetic interaction energy between microsized particles in such suspensions is much higher than the particle thermal energy.

An analytical model of chain evolution in a system of magnetizable non-Brownian particles was proposed in [8]. This model has been based on a set of kinetic equations that describe the distribution function of the number of particles in chains. The results obtained within the framework of this model are in good agreement with the data of numerical experiments.

This work has been devoted to the development of a three-dimensional computer experiment on simulating the aggregation of magnetizable particles in a viscous medium. At low concentrations, at which only linear chain-shaped clusters are observed, the simulation results are compared with the predictions of the analytical model of chain growth [8].

The work has the following structure. The basic physical concepts of the analytical model of chain growth are described in Section 2. The design of the computer experiment is discussed in Section 3. The computer experiment and analytical model are compared in Section 4. The performed computer simula- 
tion has shown that chains are only formed at particle volume concentrations of no higher than 2 or $3 \%$. At higher concentrations, dense bulk clusters are formed rather than linear chains. The results of the numerical experiment carried out for magnetic suspensions with moderate concentrations are considered in Section 5.

\section{ANALYTICAL MODEL OF CHAIN GROWTH}

The used analytical model has been described in detail in [8]. Here, we shall recall its main ideas.

The model considers a suspension of non-Brownian magnetizable particles occurring in a planar slit bounded by solid infinite parallel walls. The system is subjected to magnetic field $\mathbf{H}$ directed normal to the slit plane. It is assumed that, under the action of the field, the particles unite into linear chains oriented along the field, while aggregates of other types (branched, columnlike, etc.) are absent. According to the results of computer simulation, this approximation is justified, provided that the particle volume concentration is no higher than 2 or $3 \%$. Note that the concentrations lying in this range are quite typical of MRSs used in both laboratory studies and different fields of application.

Let us denote the number of $n$-particle chains in suspension unit volume as $g_{n}$. The goal of this work is to clarify the evolution of this function under the action of a magnetic field.

Within the framework of the accepted approximations, the evolution of an ensemble of chains is described by the following set of kinetic equations:

$$
\frac{d g_{n}}{d t}=\frac{1}{2} \sum_{k=1}^{n-1} \alpha_{n-k, k} g_{n-k} g_{k}-g_{n} \sum_{l=1}^{N-n} \alpha_{n, l} g_{l} .
$$

Here, $\alpha_{n m}$ is the rate constant of uniting $n$ - and $m$-particle chains into a chain composed of $n+m$ particles and $N$ is the maximum possible number of particles in a chain, which is determined by the slit size. Factor $1 / 2$ at the first term in Eq. (2) has been introduced in order to exclude the double account of the uniting of two chains. The upper limit in the second sum of Eq. (1) takes into account the maximum possible number of particles in a chain.

According to their physical meaning, coefficients $\alpha_{n m}$ must be symmetric with respect to the permutation of subscripts $n$ and $m$. Taking into account this circumstance, it is easy to show that, as might be expected, Eq. (1) automatically satisfies the following condition of the constancy of the total number of particles in the system:

$$
\sum_{n=1}^{N} n g_{n}=\text { const. }
$$

It is obvious that the condition const $=\varphi / V$ must be fulfilled, where $V$ is the particle volume and $\varphi$ is the overall particle volume concentration in the suspension.

A similar derivation of expressions for kinetic coefficients $\alpha_{n m}$ has been described in [8]; here, the coefficients were calculated in two approximations. In the first, simpler, approximation, variations in average interchain distance $R$ with time were ignored. Formula (4) in [8] corresponds to this approximation. The second, more awkward, approximation allowed for time variations in $R$ (formula (11) in [8]).

As was shown in [8], the simple approximation exhibits quite good agreement with the results of computer experiments for two-dimensional systems, provided that the surface concentration of particles is within 1 or $2 \%$. Below, when performing the comparison with the three-dimensional computer experiments, we use this approximation. The comparison of the computer experiments with the calculations in terms of kinetic model (1) demonstrates that the model ensures acceptable calculation accuracy, when the particle volume concentration is within 1 or $2 \%$. At higher concentrations, bulk clusters are formed and model (1), which allows for the formation of chains alone, becomes inapplicable.

Assuming that, at the initial time moment, all particles are individual, we arrive at the following initial conditions of problem (1):

$$
\left.g_{n}\right|_{t=0}=\frac{1}{V} \varphi \delta_{n 1} .
$$

Here, $\delta_{n 1}$ is the Kronecker symbol. Equations (1) and (2), which comprise kinetic coefficients $\alpha_{n m}$ that correspond to model (4) presented in [8], are easy to solve numerically.

\section{COMPUTER SIMULATION}

Within the framework of the computer model, we consider a system of similar magnetizable spherical particles. We take into account the magnetic interaction of every particle with all other particles, but ignore the hydrodynamic interchain interaction.

It is well known that, in almost all situations, the Reynolds number derived for microsized particles moving in a viscous liquid is much smaller than unity. Therefore, it is reasonable to solve the equations of motion for such particles in a noninertial approximation. Ignoring the inertia term in the Newtonian equation, we obtain the following relation for an $i$ th particle:

$$
\frac{d \mathbf{r}_{i}}{d t}=\frac{1}{3 \pi \eta d}\left(\sum_{j \neq i}\left(\mathbf{F}_{m}^{i, j}+\mathbf{F}_{s t}^{i, j}\right)\right)
$$

Here, $\mathbf{r}_{i}$ is the radius vector of the $i$ th particle; $\mathbf{F}_{m}^{i, j}$ and $\mathbf{F}_{s t}^{i, j}, \mathbf{j}$ are the forces of the magnetic and steric interaction, respectively, between the $i$ th particle and a $j$ th 


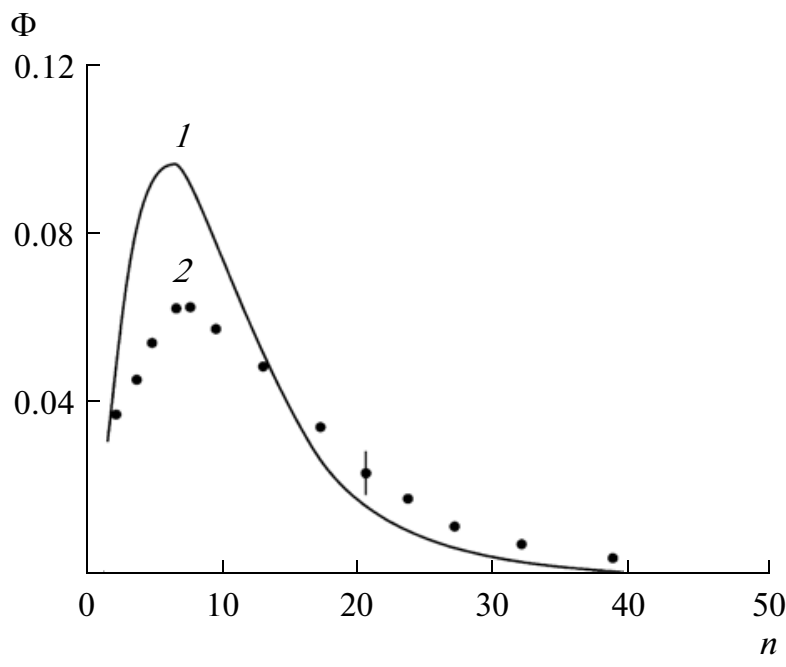

Fig. 1. Chain size distributions obtained from (1) analytical simulation and (2) numerical experiment for time moment $t=450 \mathrm{~s}$ at $\varphi=0.01$.

particle; $\eta$ is the medium viscosity; and $d$ is the particle diameter.

Approximation (3) implies that a particle occurring in a chain is subjected to the same viscous drag as an individual one. In this approximation, the hydrodynamic mobility of an aggregate composed of number $n$ of strongly interacting particles is proportional to $1 / n$. This approximation is frequently used in computer models of magnetic suspensions (see, e.g., [9-11]). In particular, it was applied in our previous calculations [8]. The use of these estimates of the mobility is justified by the fact that the mobility of a chain cannot be calculated analytically because of its complex shape. Determination of the mobility by numerical solution of the Navier-Stokes equation also encounters great difficulties. At the same time, the asymptotic formulas available for the mobility of strongly elongated axially symmetric bodies (see, e.g., [12]) with a major-tominor axial ratio equal to $n$ yield a dependence of the mobility on this parameter close to $1 / n$. This circumstance allows us to consider Eqs. (3) to be a quite justified approximation.

In the approximation of the dipole-dipole interaction, the radial and tangential components of the magnetic force may be written as follows:

$$
\begin{aligned}
F_{m, r}^{i, j} & =\frac{3 m_{i} m_{j}}{4 \pi \mu_{0}} \frac{1-3 \cos ^{2} \theta}{r_{i, j}^{4}}, \\
F_{m, \theta}^{i, j} & =-\frac{3 m_{i} m_{j}}{4 \pi \mu_{0}} \frac{2 \sin \theta \cos \theta}{r_{i, j}^{4}},
\end{aligned}
$$

Here, $r_{i j}$ is the distance between the centers of the particles, $m_{i}$ and $m_{j}$ are their magnetic moments, $\theta$ is the angle between imposed field $\mathbf{H}$ and radius vector $\mathbf{r}_{i j}$, and $\mu_{0}$ is the magnetic permeability of vacuum.
In order to maximally simplify the calculations, we ignore the mutual magnetization of particles in a chain. Note that this simplification is often applied in the theory of magnetic suspensions [1]. In the approximation of the linear particle magnetization, i.e., assuming that magnetic permeability $\mu_{p}$ is independent of the magnetic field, we have [13] the following:

$$
m=\frac{\pi \mu_{0} d^{3}}{2} \frac{\mu_{p}-1}{\mu_{p}+2} H
$$

The short-range forces of interparticle steric interaction were simulated as follows. At mutual overlapping of particles (at $r_{i j} \leq d$ ), the software package used in the experiment generated an interparticle repulsive force that forced the particles to spread at distance $d$ over one spatial step of calculations. Therewith, at distances $r_{i j} \leq d$, the magnetic attraction was assumed to be equal to zero. As soon as the distance between the centers of the particles became larger than or equal to $d$, the repulsive force was nullified, while the attractive force was restored. This procedure enabled us to substantially cut the duration of the computer experiment to compare with the approximations implying a smooth repulsive force, which are traditionally used to simulate the steric effects.

\section{CHAINS IN LOW-CONCENTRATED SUSPENSIONS}

Several thousand particles were used in our computer experiment program. Equations (3) and (4) were solved for all particles of the system. At the onset of the experiment, individual particles were randomly distributed over a cell with edge sizes equal to 50 particle diameters. The time step of the calculations was selected in a manner such that the displacement of a particle over one step was no larger than $d / 20$. The calculation results were averaged over a few realizations of the initial conditions.

Some results of our computer calculations and their comparison with the calculations performed in terms of the analytical model are illustrated in Figs. 1 and 2, where the size distributions of the relative numbers of particles in the chains $\Phi=\frac{n g_{n} V}{\varphi}$ are presented. The calculations were implemented at parameters $\eta=$ $20 \mathrm{~Pa}$ s and $H=16.5 \mathrm{mT}$, which corresponded to real MRSs and experiments.

The data obtained using the analytical model and computer experiment are in good agreement with each other. Hence, the model described by Eqs. (1) can be used for approximate description of the kinetics of chain growth in low-concentrated MRSs, when the formation of clusters with other shapes may be ignored.

As was mentioned above, the data obtained in computer and laboratory experiments on the kinetics of chain-shaped structure growth in magnetic suspen- 


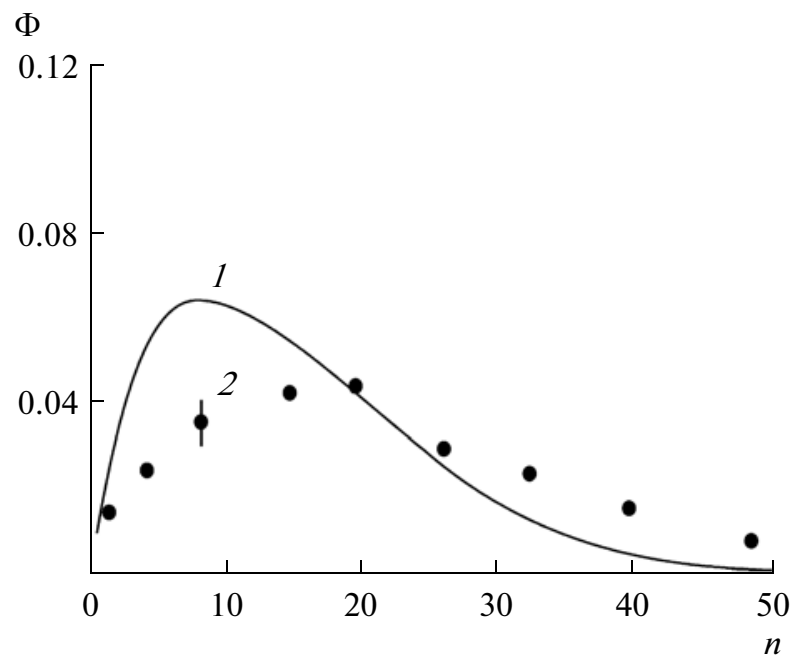

Fig. 2. The same as in Fig. 1 for time moment $t=900 \mathrm{~s}$.

sions are frequently approximated by the $\langle n\rangle \propto t^{k}$ dependence. Figure 3 illustrates the time dependences of $\langle n\rangle$ determined in computer experiments at four concentrations $\varphi$ and the approximations of these dependences by the function

$$
\langle n\rangle=1+C t^{k},
$$

which takes into account that, at $t=0$, only monomers are present in the system; i.e., $\langle n\rangle=1$. Parameters $C$ and $k$ were selected from the data of the computer experiment by the method of least squares.

The magnetic attraction of particles to vessel walls was not taken into account in the above results. This is justified, provided that the walls are made of a diamagnetic material. However, walls made of paramagnetic metals are used in some experiments. Magnetized particles are attracted to such walls so as to lose their mobility and the possibility of moving in directions both perpendicular to and along the walls. The lateral displacement is blocked by the friction between the particles and walls.

In the computer simulation, we took into account the interaction with walls by introducing image forces (see, e.g., [13]). If the distance between a wall and the center of a particle was smaller than $d(1+d / 20) / 2$, which corresponded to the thickness of a rough layer on the surface of vessel walls, the displacement of particles along the walls was blocked.

The structure of chains formed with a concomitant attraction of particles to slit walls is shown in Fig. 4. Some of the particles adhere directly to the walls, and chains are bonded to them. These chains lose their mobility, and the process of suspension structuring slows down. Note that aggregates depicted in this figure represent linear chains. In the figure, some of them are projected onto one another and resemble a bulk aggregate.

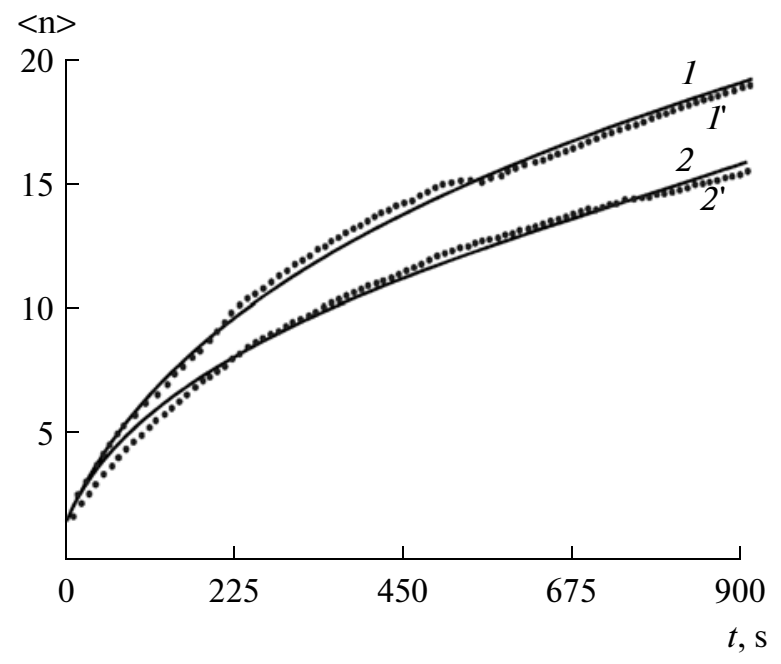

Fig. 3. Dependences of the average chain size in the $(1,1)$ absence and $(2,2)$ presence of attraction to the walls: (1, $2)$ calculation by Eq. $(6)$ and $(1,2)$ numerical simulation; $\varphi=0.02$ and slit thickness is $50 \mathrm{~d}$.

The dependence of average number $\langle n\rangle$ of particles in a chain on time $t$ for particles attracted to slit walls is also shown in Fig. 3 together with the approximating curve plotted in accordance with expression (6). The values of fitting parameters $C$ and $k$ selected using the method of least squares are presented in Table 1 for both cases under consideration. It can be seen that exponent $k$ is not a universal constant value. It depends (although not too strongly) on particle volume concentration $\varphi$.

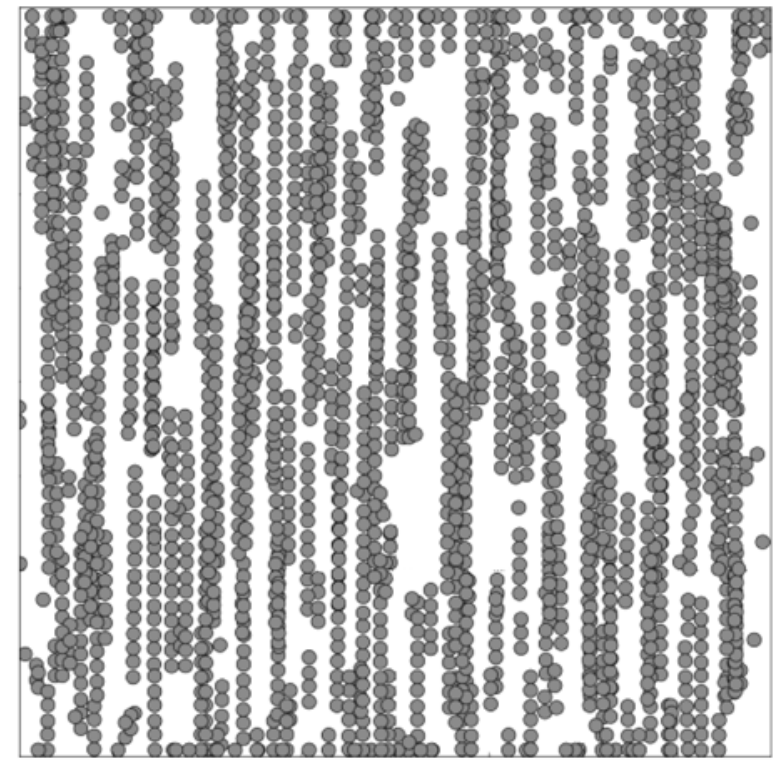

Fig. 4. The structure obtained in the numerical experiment at time moment $t=450 \mathrm{~s}$ in the presence of particle attraction to the walls; $\varphi=0.01$. 
Table 1. Dependence of fitting parameters $k$ and $C$ on particle volume concentration $\varphi$ in the absence of attraction of particles to slit walls

\begin{tabular}{l|c|c}
\hline \multicolumn{1}{c|}{$\varphi$} & $k$ & $C$ \\
\hline 0.005 & 0.753 & 0.0224 \\
0.0075 & 0.807 & 0.0267 \\
0.01 & 0.775 & 0.0492 \\
0.0125 & 0.729 & 0.0492 \\
0.015 & 0.707 & 0.116 \\
0.02 & 0.631 & 0.252 \\
\hline
\end{tabular}

Table 2. The same as in Table 1 for the case of particle attraction to slit boundaries

\begin{tabular}{l|l|l}
\hline \multicolumn{1}{c|}{$\varphi$} & $k$ & \multicolumn{1}{|c}{$C$} \\
\hline 0.005 & 0.707 & 0.0267 \\
0.0075 & 0.718 & 0.0418 \\
0.01 & 0.678 & 0.0740 \\
0.0125 & 0.644 & 0.119 \\
0.015 & 0.606 & 0.184 \\
0.02 & 0.546 & 0.355 \\
\hline
\end{tabular}

Comparison of the dependences in Fig. 3 and the values of exponent $k$ listed in Tables 1 and 2 shows that the attraction of the particles to the slit boundaries decelerates the aggregation of the suspension. Indeed, the average length of the chains at the same time moment is markedly smaller when the particles are

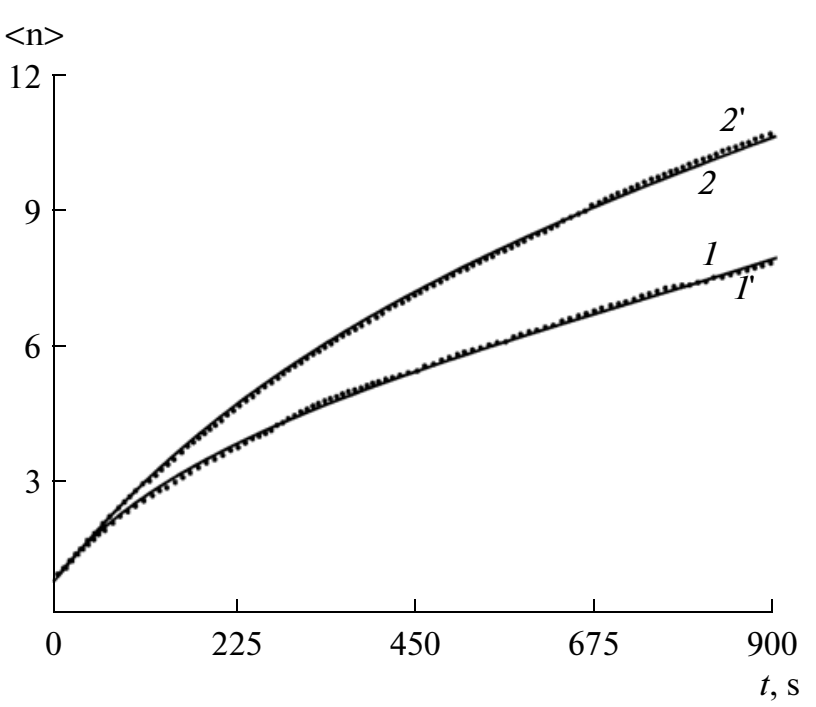

Fig. 5. Time dependences of the average chain size obtained for slits with sizes of $(1,1) 25 d$ and $(2,2) 50 d$ by $(1,2)$ calculation through Eq. (7) at (1) $C=0.15$ and $k=$ 0.55 and (2) $C=0.12$ and $k=0.626$ and $\left(1^{\prime}, 2^{\prime}\right)$ numerical calculation; $\varphi=0.01$. attracted to the walls than in the absence of the attraction.

The size and shape of the suspension-containing cell may affect the rate of particle aggregation. This effect is caused by, first, the dependence of the internal Maxwell field on the shape of a sample and, second, merely geometrical reasons, which change the conditions for the coalescence of chains upon changes in their sizes. Figure 5 shows the time dependences of the average chain sizes for slits of different thicknesses. It can be seen that an increase in the slit thickness accelerates the aggregation process.

\section{MODERATELY CONCENTRATED SUSPENSIONS. BULK AGGREGATES}

As was mentioned above, at particle volume concentrations of higher than 2-3\%, particles coalesce into dense bulk aggregates. The images of these aggregates in a system with an overall particle volume concentration of $10 \%$ are shown in Fig. 6 . The performed computer experiments have demonstrated that an increase in the slit thickness accelerates the aggregation process and stimulates the formation of bulk clusters. While only chain-shaped aggregates were observed in relatively thin layers, bulk aggregates begin to be formed with a rise in the slit thickness, other system parameters being equal.

Figure 6 provides information on the peculiarities of the spatial organization of bulk clusters (the field is oriented in parallel with the plane of the figure) in a slit with sizes of $50 d \times 50 d \times 10 d$. At the first stage, bulk structures extended along the field are formed in the initial suspension having a uniform particle distribution (compare Figs. 6a and 6b). At the second stage, a slow evolution of these structures, in particular, their densification, takes place (the intercluster gaps are somewhat larger in Fig. 6c than in Fig. 6b).

Figure 7 illustrates the structure of the same systems as those in Fig. 6, but observed along the magnetic field (the field is oriented normal to the plane of the figure). It is of interest that the clusters have an irregular shape.

Irregular shapes of the bulk clusters were observed in the computer [9] and laboratory [14] experiments with suspensions of magnetic non-Brownian particles. Note that, in thin layers of colloidal nanodisperse ferrofluids, the particles of which are involved in intense Brownian motion, dense domains with an almost regular cylindrical shape are commonly observed (see, e.g., $[15,16])$. A difference of principle between dense domains formed in thin layers of magnetorheological suspensions, on the one hand, and ferrofluids, on the other hand, consists in the fact that, due to the Brownian motion, the particles of the latter reach a thermodynamically equilibrium state over a short time of fractions of a second or a few seconds. Therefore, dense quasi-cylindrical domains are rapidly formed in such systems, the sizes of the domains being deter- 
(a)

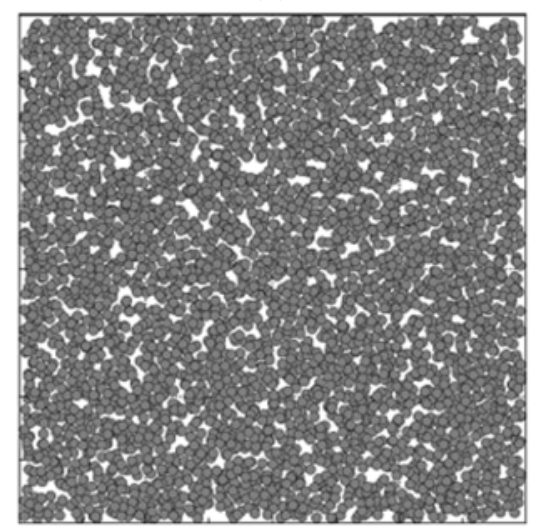

(b)

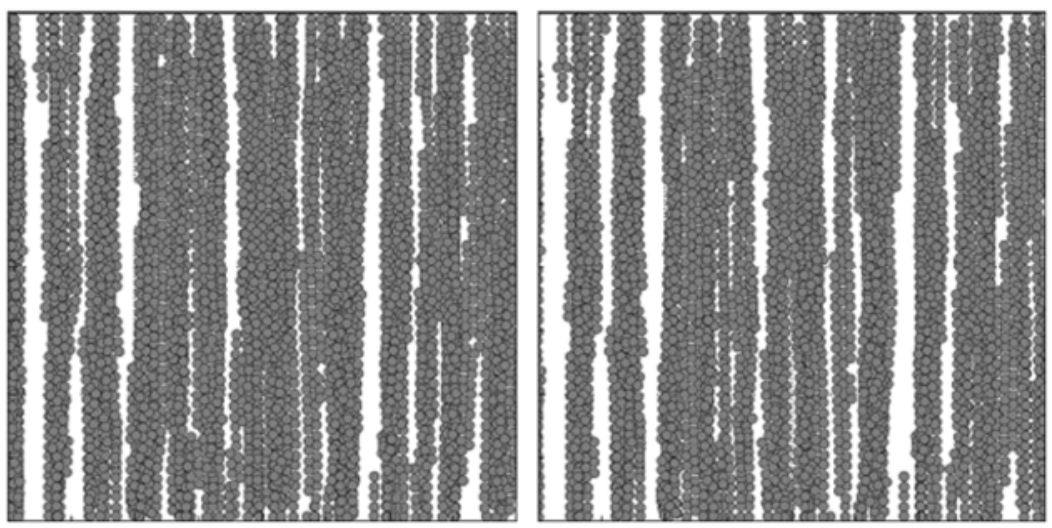

Fig. 6. The character of time variations in the structure of magnetic fluid: (a) initial time moment and time moments $t=(\mathrm{b}) 450$ and (c) $900 \mathrm{~s}$ after the field is switched on; the view in the direction normal to the field; $\varphi=0.1$.

(a)

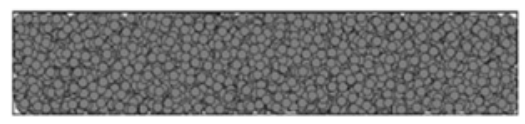

(b)

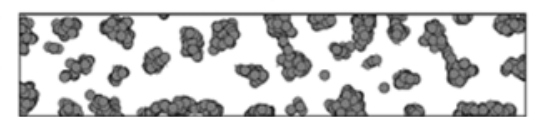

(c)

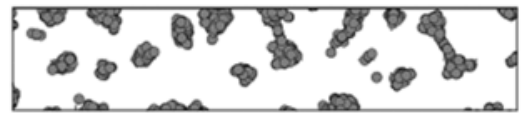

Fig. 7. The same as in Fig. 6 for the view in the direction of the field.

mined by the balance between the effects of a demagnetizing field, which tend to decrease the domain thickness as much as possible, and the effects of the surface tension, which tend to minimize the total interfacial area [17].

At the same time, non-Brownian microsized particles of magnetorheological suspensions are frozen in random local energy minima, the escape from which and the passage to the global minima requires a very long time, which frequently exceeds a reasonable time of observations. This results in the appearance of domains with a "frozen" irregular structure, which is observed in laboratory and numerical experiments.

\section{CONCLUSIONS}

The results of three-dimensional computer simulation have been presented for the formation kinetics of chain-shaped aggregates in suspensions of nonBrownian magnetizable particles. When the particle volume concentration is no higher than $2-3 \%$, only linear chain-shaped aggregates are formed in a system. In this case, the evolution of the system may, as a first approximation, be described by the simple kinetic model proposed in [8]. The time dependence of the average number of particles in a chain may be described by a power law, with the exponent being dependent on the particle concentration. An enlargement of a slit in the direction of the field accelerates the growth of the chain length due to both the enhancement of the macroscopic Maxwell field in the slit and the geometric reasons that facilitate the chain aggregation.

When the particle concentration in a suspension is higher than $2-3 \%$, particles coalesce into bulk clusters extended along the field. In the plane of the slit, these clusters have an irregular shape, which can be explained by the "frozen state" of particles in local energy minima, which prevents the system from reaching thermodynamic equilibrium within a reasonable time.

\section{ACKNOWLEDGMENTS}

This work was supported by the Russian Foundation for Basic Research (projects nos. 10-01-96002Ural, 10-02-96001-Ural, 10-02-00034, and 12-0100132) and the Scientific Program of the Ministry of Education of the Russian Federation (project no. 2.1267.2011).

\section{REFERENCES}

1. Bossis, G., Volkova, O., and Lacis, S., in Ferrofluids, Magnetically Controllable Fluids and Their Applications, Odenbach, S., Ed., Berlin: Springer, 2002, p. 202.

2. Bayat, N., Nethe, A., Guldbakke, J.M., et al., in Colloidal Magnetic Fluids, Odenbach, S., Ed., Berlin: Springer, 2009, p. 359.

3. See, H. and Doi, M., J. Phys. Soc. Jpn., 1991, vol. 60, p. 2778. 
4. Climent, E., Maxey, M.R., and Karniadakis, G.E., Langmuir, 2004, vol. 20, p. 507.

5. Miguel, M.C. and Satorras, R.P., Phys. Rev. E: Stat. Phys., Plasmas, Fluids, Relat. Interdiscip. Top., 1999, vol. 59, p. 826.

6. Dominguez-Garcia, P., Melle, S., Pastor, J.M., and Rubio, M.A., Phys. Rev. E: Stat. Phys., Plasmas, Fluids, Relat. Interdiscip. Top., 2007, vol. 76, p. 051403.

7. Bossis, G., Metayer, C., and Zubarev, A., Phys. Rev. E: Stat. Phys., Plasmas, Fluids, Relat. Interdiscip. Top., 2007, vol. 76, p. 041401.

8. Bossis, G., Iskakova, L., Kostenko, V., and Zubarev, A., Physica A (Amsterdam), 2011, vol. 390, p. 2655.

9. Mohebi, M. and Liu, J., Phys. Rev. E: Stat. Phys., Plasmas, Fluids, Relat. Interdiscip. Top., 1996, vol. 54, p. 5407.
10. Tan, Z-J., Zou, X-W., Zhang, W-B., and Jin, Z.-Z., Phys. Rev. E: Stat. Phys., Plasmas, Fluids, Relat. Interdiscip. Top., 1999, vol. 59, p. 3177.

11. Ilg, P. and Odenbach, S., in Colloidal Magnetic Fluids, Odenbach, S., Ed., Berlin: Springer, 2009, p. 249.

12. Van de Ven, T.G.M., Colloidal Hydrodynamics, New York: Academic, 1989.

13. Landau, L., Lifshitz, E., and Pitaevski, L., Electrodynamics of Continuous Media, Oxford: Pergamon, 1984.

14. Lopez-Lopez, M.T., Duran, J.D.G., Rodriguez-Arco, L., Zubarev A., and Iskakova, L., J. Appl. Phys., 2010, vol. 108, p. 083503.

15. Bacri, J.C. and Salin, D., J. Magn. Magn. Mater., 1983, vol. 9, p. 48.

16. Blums, E., Cebers, A., and Majorov, M.M., Magnetic Fluids, Berlin: De Gruyter, 1997.

17. Buyevich, Yu.A. and Zubarev, A.Yu., J. Phys. II, 1993, vol. 3, p. 1633. 